\title{
Strategies for Multiple Sequence Alignment
}

BioTechniques 32:572-591 (March 2002)

\section{Hugh B. Nicholas Jr., Alexan- der J. Ropelewski, and David W. Deerfield II \\ Carnegie Mellon University, Pittsburgh, PA, USA}

\begin{abstract}
We present an overview of multiple sequence alignments to outline the practical consequences for the choices among different techniques and parameters. We begin with a discussion of the scoring methods for quantifying the quality of a multiple sequence alignment, followed by a discussion of the algorithms implemented within a variety of multiple sequence alignment programs. We also discuss additional alignment details such as gap penalty and distance metrics. The paper concludes with a discussion on how to improve alignment quality and the limitations of the techniques described in this paper.
\end{abstract}

\section{INTRODUCTION}

Just a few years ago, molecular biologists and protein biochemists were pleased when their database search resulted in a small set of well-characterized, homologous sequences that provided reliable leads to the biochemical function and physiological role of their gene or gene product. The recent explosion of sequence data has greatly expanded these sets of homologues to include many diverse organisms, each of which may be represented by multiple genes or gene products. The biochemical function and physiological roles of these extended sets of sequence homologues, often referred to as gene or protein superfamilies, may have diverged significantly. An estimate of the detailed evolutionary history of a set of homologous sequences from which insights into these differing biochemical functions and physiological roles can be derived is implicit in, and a major motivation for, the creation of a multiple sequence alignment.

Homologous sequences trace their parentage to a single gene, the common ancestor. The evolutionary relationship (Figure 1) can consist of only mutations of the gene (orthologous sequences) or an evolutionary process that also includes gene duplication (paralogous sequences). Generally, orthologous sequences will have similar or identical physiological roles, while paralogs will often have different physiological roles. An example would be the globins, where all globins transport oxygen, but myoglobin is a monomer that transports oxygen within the cell, while hemoglobin is an $\alpha, \beta$ tetramer that transports oxygen in the bloodstream. The orthologous sequences are usually under similar evolutionary constraints even when in different organisms, whereas paralogous sequences are potentially under very different evolutionary constraints because of their different physiologic roles. Note that the distinction between orthologous and paralogous genes becomes much less clear in highly divergent organisms that face very different evolutionary constraints (28).

The orthologous and paralogous sequences will usually have a series of highly conserved residues that are essential for defining either the overall structure or the function of the entire sequence family. Ideally, the multiple sequence alignment should also describe the conserved nature of orthologous sequences while differentiating the paralogous sequences. That is, the patterns observed in a multiple sequence alignment should reflect the evolutionary history that relates the sequences.

It should be clearly understood that all multiple sequence alignment programs assume that the sequences are actually related. That means that each program will compute an alignment given a set of sequences, regardless of whether the sequences are actually related through a common ancestor. Demonstrating that the sequences are actually related is the responsibility of the investigator. Failure to properly determine that the sequences are related through evolution can lead to incorrect results and unfortunate consequences $(32,35)$. A multiple sequence alignment program aligns several sequences in ac- 
cordance with a model of evolution, as expressed in its method for scoring the alignment of different residues and the introduction of gaps. Available algorithms make different assumptions and apply different approximations in accumulating the score over the entire alignment. Each of these different assumptions and approximations can have a profound effect on the resulting multiple sequence alignment. In this paper, we describe the different assumptions and approximations made by some of the more widely used multiple sequence alignment algorithms and provide guidance on how to recognize when these assumptions and approximations have introduced artifacts.

In the following discussion, we have conceptually divided the multiple sequence alignment problems into two parts. The first part describes the various methods for scoring multiple sequence alignments and the relevance of the scoring method to a biological branching process. The second part describes in detail the various strategies that researchers have implemented for optimizing a multiple sequence alignment, given a scoring method. Included within both of these sections is a discussion on the artifacts introduced into multiple sequence alignments because of shortcomings of the scoring method and implementation strategies. We close with a discussion on other relevant multiple sequence alignment topics, including gap penalties, distance measures, improving alignment quality, and the relative quality of the automatic alignment programs.

\section{SCORING METHODS}

The scoring method defines the objective function used for computing the score of the multiple sequence alignment. This score, how it is computed, and how well this computation reflects the underlying evolutionary processes that gave rise to the sequences being aligned are critical in determining how accurately the resulting alignment reproduces the evolutionary history of the sequences. Thus, the scoring method should reflect the biological mechanism for creating the present-day sequences and be simple and easy to interpret.
Several scoring methods have been used to score the multiple sequence alignment: those based on an explicit tree $(43,54)$, a sum-of-pairs measure $(25)$, and a host of other methods $(16,37,38)$. All methods attempt to minimize the number of evolutionary events required to describe the divergence of the observed sequences. The actual course of evolution may, of course, have been different and included more evolutionary events. Thus, the actual course of evolution for highly diverged sequences could substantially deviate from the minimum course assumed during the creation of the alignment.

\section{Evolutionary Branching Process}

Probably the most biologically relevant method to score a multiple sequence alignment is based on an evolutionary branching process that is implied by the alignment. The most common evolutionary branching process is a simple unrooted tree (Figure $2 \mathrm{~A}$ ) in which the actual ancestral sequence (the root) is not known, but the relative distances and connectivities between sequences are known. The second example is a star (Figure 2B) in which it is assumed that all sequences descend from the root. The star is a special instance of a rooted tree and is appropriate when one sequence gives rise to many descendants, such as with viruses or epidemiological studies.

There are several approaches (31, 41) for creating a tree to use in scoring an alignment, including measures based on distance, parsimony, or a statistical model such as maximum likelihood. Distance methods for determining trees compute the distance between all pairs of sequences by either a simple pairwise procedure or by a more com plicated approach that computes a degree of divergence per alignment position and then determines the tree that best fits these distances. The parsimony measure scoring method involves directly reconstructing the ancestral sequence at each node and then counting the changes along each leg. This often involves the creation and evaluation of a large number of possible trees. The maximum likelihood approach integrates a large probability function across the entire tree and sequence length, including the probability for all mutations, along with insertions and deletions, which makes this method the most computationally expensive approach. Given a method of producing a tree from an alignment, each alignment implies a specific tree (i.e., there is a circular relationship among the actual alignment, the method used to generate the tree, and the resulting tree). Two tree-producing methods are not guaranteed to produce the same tree from the same alignment; conversely, it is possible that two different tree-producing methods that operate on two different alignments will compute the same tree.

Overall, the optimization of multiple sequence alignment based on an evolutionary branching mechanism is almost

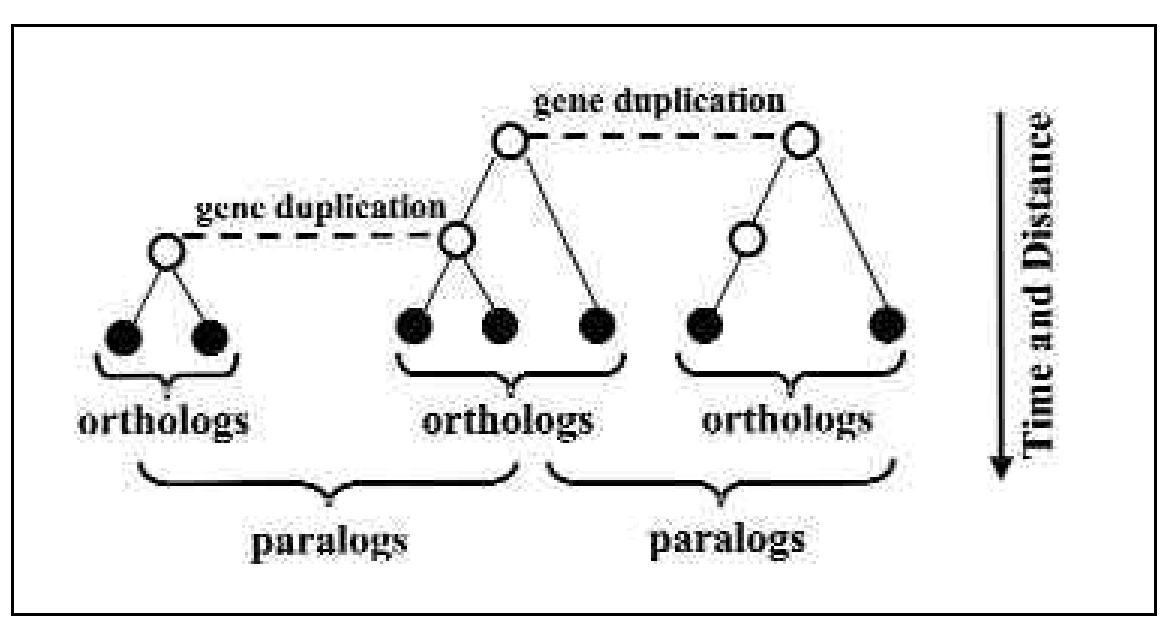

Figure 1. A hypothetical, idealized family tree that demonstrates the difference between orthologous and paralogous sequences. Open circles represent ancestral sequences, and closed cirles represent present-day sequences. In a number of sequence families, the orthologous sub-trees would overlap. 
computationally intractable (43). Only a few programs are currently available (54), and these programs are not widely used by the general biology community. Because of the computational complexity of these approaches, there is little information on which to discuss the actual quality of the resulting alignment.

\section{Sum-of-Pairs}

The sum-of-pairs method is an extension of the scoring method used in pairwise alignments $(30,44)$ and is the most popular scoring method because of its relative speed and robustness. The speed advantage is primarily because the sum-of-pairs method does not require a tree. The sum-of-pairs score is computed by evaluating the distance between every pair of residues in a single aligned column (Figure 2C). The principal defect of the sum-of-pairs scoring method is that it overcounts the internal branches of a tree relative to the external branches, which results in alignments that yield trees that have short internal branches and long terminal branches and are highly congested. Essentially, the tree looks more like a bush. An alignment optimized using the sum-of-pairs method will tend to have gaps aligned with each other in a small number of columns, thus underestimating the number of insertion and deletion events in the evolutionary history of the family of sequences.

Traveling salesman scoring. Because of the computational complexity of the evolutionary branching process approach and the limitations of the sum-of-pairs approach, several groups are developing alternative approaches to score multiple sequence alignments. One of the more promising approaches is based on the traveling salesman algorithm. Gonnet and co-workers (16) have shown that a traveling salesman problem can be mapped onto a minimal spanning tree (Figure 2D), with the solution being twice the sum of the branches in the minimal spanning tree. Thus, the method captures distances between pairs of sequences without overweighting the interior branches of the prospective evolutionary tree that is relating the sequences. This approach is available in the Darwin sequence analysis system (15).
Entropy scoring. Minimum entropy scoring has been suggested by several mathematically and statistically oriented investigators (10). Entropy is computed for each column in the alignment, and these computations are summed to give an overall score. The entropy (as the term is defined in entropy scoring) for a single column is the sum over all kinds of residue of the products of the fraction of each residue in the column times the log of that fraction. A column receives a score of zero when all of the residues in the column are the same kind and the highest score when there are equal numbers of all of the possible kinds of residues in the column (i.e., including gap characters, which are treated as another kind of sequence residue). Entropy scoring captures the degree of conservation encapsulated within an alignment but is difficult to relate to the phylogenetic aspects of the alignment because there is no underlying evolutionary model.

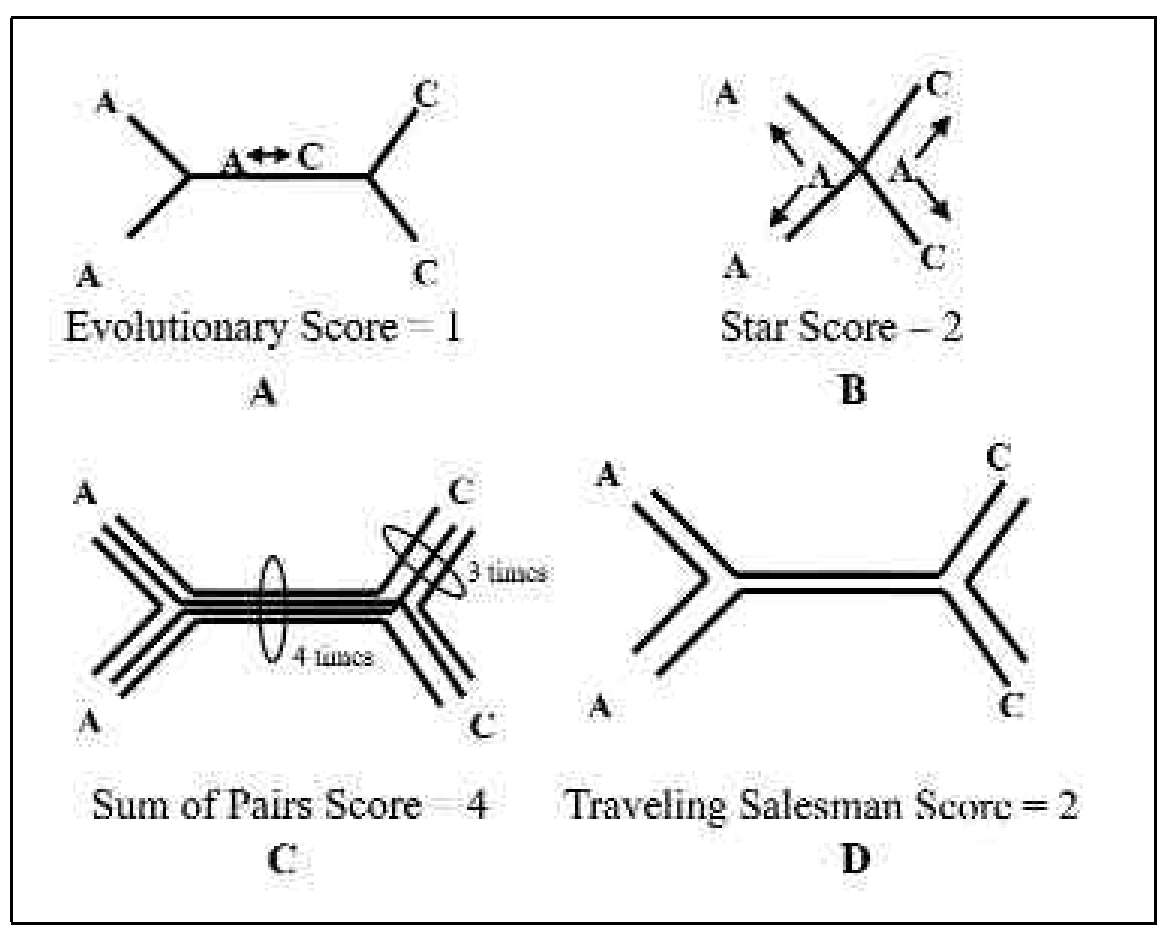

Figure 2. Different scoring methods and the score that each method would yield for four sequences. (A) Scoring along the branches of an unrooted tree. The single evolutionary event, the change from A to $\mathrm{C}$ or from $\mathrm{C}$ to $\mathrm{A}$, as is shown above the central branch of the tree, is the only event that results in a score for this method. This results in a score of one. (B) The same change either from A to Cor from $\mathrm{C}$ to $\mathrm{A}$ occurs along two of the branches that lead from the center of the star tree. These two changes are enough to yield the observed sequences in this case; thus, the score is two. (C) Each independent path from every sequence character to the others is shown and results in a count of one added to the score when the two sequences are different and a count of zero added when the sequences are the same. This yields a total score of four. (D) As in part C, a path is shown between each pair of sequences that contributes either a zero or one to the total score. These paths yield a total score of two. 
extended by examining the consistency of the pairwise alignments among all of the triplets of the sequences in the data. Users may specify additional alignments, such as structural alignments, to use in developing the scores.

During this score development stage, the T-Coffee procedure examines and accounts for many of the situations in the scores that normally lead to problems and errors for other progressive pairwise multiple sequence alignment procedures. In particular, the local alignments used by T-Coffee are expected to identify highly conserved regions that are found in all or many of the sequences in the data. These regions will be given very favorable scores when they are aligned with each other in the final stages of the process and, thus, are likely to be part of the final alignment.

The DbClustal (53) program that computes a multiple sequence align- ment from the query sequence and all of its full-length homologues discovered in a BLAST database search take a sim ilar approach. DbClustal computes scores for "anchor regions" for all pairs of sequences in the alignment set, based on the results of the initial BLAST search. These anchor region scores, computed as described for the BLAST post-processing program Ballast (39), are used to supplement the normal ClustalW (49) alignment process.

\section{Comments About Scoring Methods}

In theory, the optimization of a multiple sequence alignment based on a tree scoring method is the most scientifically satisfying approach and an active area of research $(21,54,56)$. The attraction is that the optimization is ultimately based on an explicit evolutionary model. However, the development of a program that fully incorpo- rates this scoring method with an optimization scheme that can run in a reasonable time has proven to be an elusive goal, with no program currently in use by the general biology community, although evolutionary biologists sometimes find the time and expense worthwhile (55). The continued improvement in available computational resources coupled with algorithm development $(21,56)$ could lead to the development of a general tree optimization program in the future. While it is always preferable to use methods firm ly grounded in theoretically rigorous evolutionary models, our lack of experience with such models leaves us uncertain about how big a change these methods would make in the best alignments created by currently available methods, generally rooted in the sumof-pairs approach. While the sum-ofpairs score is not theoretically rigorous in an evolutionary sense, it has lead to 
practical sequence alignment methods that have yielded scientifically useful results. Further, sum-of-pairs methods have provided a useful starting point for extensive research into multiple sequence alignment problems.

\section{IMPLEMENTATION STRATEGIES OF THE SUM- OF-PAIRS SCORING METHOD}

The previously described scoring methods provide a procedure to evaluate the quality of a multiple sequence alignment and, given a series of multiple sequence alignments, can identify the best. Thus, the second half of the problem is to use the knowledge incorporated within the scoring method to find an optimum alignment. Ideally, one would like to couple efficiently the procedure to find an optimal alignment with the scoring method.

There are three conceptual approaches toward finding the optimal multiple sequence alignment: $(i)$ to find a solution using all of the data simultaneously $(25,36,40)$; (ii) to define heuristics that divide the problem into smaller, more

Table 1. Main Steps in the MSA Alignment Algorithm

1. Determine the optimal pairwise alignment for each pair of sequences.

2. Perform a fast multiple sequence alignment (see PPA below) of all of the sequences and extract the pairwise alignments from this multiple sequence alignment.

3. For each pair of sequences, use the optimal and extracted pairwise alignments to define the restricted alignment space defined by the difference in the two alignment scores for this pair of sequences (Figure 3, left). This includes the area covering all alignments that score no more than the score for the extracted pairwise alignment and generally extends somewhat beyond that.

4. Project the restricted pairwise alignment spaces into the multidimensional alignment space to define the restricted hyper-volume of the multidimensional space to determine the best multiple sequence alignment (Figure 3, right). The greater the overall sequence similarity, the smaller the restricted alignment space is.

5. Use dynamic programming to compute the value for all of the cells within the restricted alignment space. Cells on the boundaries require special treatment, and the restricted alignment space can be slightly expanded on the fly.

6. Backtrack through the restricted alignment space to recover the best alignment. The result is a minimum distance alignment.

tractable problems whose solutions can be combined to provide the complete solution $(4,7,8,12)$; or $(i i i)$ to start with a low-quality alignment and improve it in a defined series of iterative steps $(17,22,36)$. The simultaneous methods examine all of the data at once to find the best alignment, which often results in a process that is computationally challenging in both CPU time and mem ory required. Thus, heuristics and iterative approaches have been developed to reduce the overall computational expense (usually memory requirements)
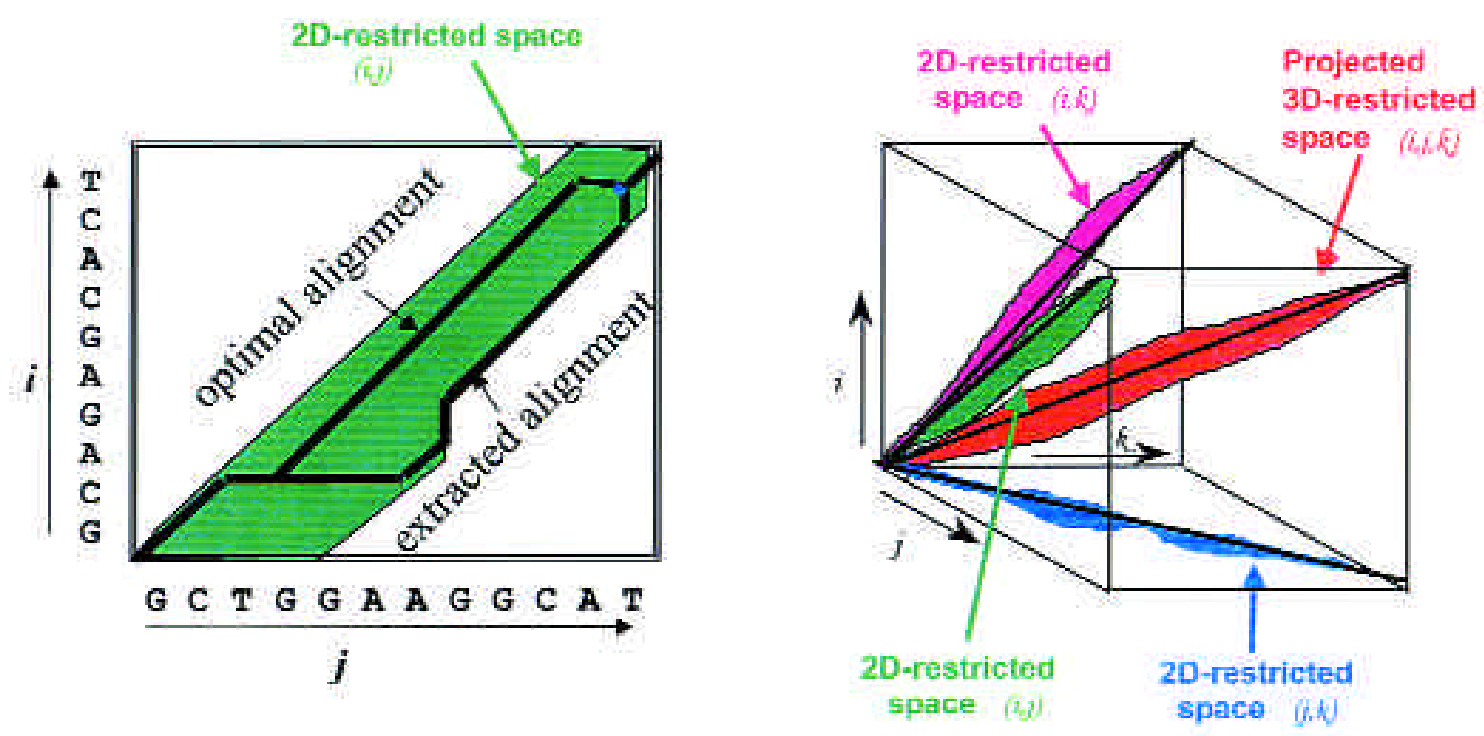

Figure 3. An example of how the MSA program would restrict the alignment space for the multiple sequence alignment of three sequences $(i, j, k)$. On the left is the planar alignment space for one pair of sequences. The heavy black line to the upper left is the alignment path graph for the optimal global pairwise alignment, and the heavy black line to the lower right is the alignment path graph for the pairwise alignment extracted from a multiple sequence alignment of two sequences, $i$ and $j$. The green-colored area represents the 2D-restricted alignment space used in the MSA algorithm, and it covers all of the area that contains alignments that score no more than the score for the pairwise alignment extracted from the heuristic multiple sequence alignment. Generally, the area is enlarged somewhat beyond this minimum. On the right is the projection of the three pairwise (2D)-restricted alignment spaces to define the projected 3D-restricted space used for computing the actual alignment. 
while trying to compute the same alignment as provided by more computationally intensive algorithms. The heuristics suffer from local minima problems caused by combining partial solutions into a complete solution, while the iterative approaches tend to find the best alignment with potentially large (and unpredictable) CPU time requirements.

The following section describes the strategies for finding a multiple sequence alignment for the widely used programs. The emphasis is on the strengths and weaknesses of each approach and on how to identify the artifacts that each method may introduce into the multiple sequence alignments. We also include a small discussion on other approaches, especially Hidden Markov Models (HMMs), because of the attention that HMMs have recently received $(3,11)$.

\section{Multidimensional Dynamic Programming Technique}

Multidimensional dynamic program ming is the most computationally intensive technique to minimize the sum-ofpairs scores but, given a scoring method, is guaranteed to provide the global minimum. There are a number of implementations of the dynamic programming technique that have been applied to pairwise sequence comparison: the Needleman-Wunsch (30), and its equivalent formulation using a distance measure first presented by Sellers (44), for global alignment; the Smith-Waterman (46) for local alignment; and the Seller (45) quasi-global alignment (see Reference 34 for a more complete description). Since the multiple sequence alignment programs discussed here assume that the entire sequence is to be aligned, we used the global Sellers equivalent for the Needleman-Wunsch implementation of the dynamic programming technique. The multidimensional implementation is conceptually identical to the pairwise implementation (1). The computational requirements for a full multidimensional alignment, both in CPU time and memory, scale as the product of the length of the sequences. Thus, the computational requirements can quickly become prohibitive beyond a small number of short sequences.

David Lipman's group at the NCBI
(25) has developed a program, MSA, that makes multidimensional program ming feasible for a moderate number of sequences by examining only a small, carefully selected portion of the total possible alignment space. The program finds the best alignment within the examined region of the alignment space. If the region of alignment space exam ined contains the global minimum distance alignment, which may often be the case given the procedure by which the region is selected, then the program will find it. Table 1 describes the overall approach.

The key insight by Lipman et al. (25) is that the sum-of-pairs score for a multiple sequence alignment cannot be lower than the sum of the alignment scores for the optimal alignments for each pair of sequences. The optimal pairwise alignment score will be the lower bound on the score for the pairwise alignment extracted from the nonoptimal, fast, heuristic multiple sequence alignment. In other words, we expect that the score for the pair of sequences (as aligned in the globally optimal alignment) will be between the score for the optimal pairwise alignment and the score for the pairwise alignment extracted from the fast, heuristic multiple sequence alignment. Thus, the optimal multiple sequence alignment score should be bounded by the scores of the pairwise and extracted alignments. Gupta et al. (19) have reported several improvements on the original MSA code.

The MSA program does a reason- ably good job of defining the restricted alignment space, and if the true global minimum alignment is within this space, then the program will find this alignment. In practice, MSA works well on a reasonable number of medium-length proteins. For example, on a mini-supercomputer with $4 \mathrm{~GB}$ of physical memory, MSA can optimally align 20 phospholipase A2 sequences (approximately 130 residues), 14 highly diverse cytochrome $\mathrm{C}$ sequences (approximately 110 residues), six moderately diverged aspartyl proteases (approximately 350 residues), or eight moderately diverged lipid-binding proteins (approximately 480 residues). Each of these problems approaches the limit of what can be optimally aligned by the MSA program, which is related to the number, length, and diversity of the sequences.

\section{Heuristic for the Multidimensional Dynamic Programming Technique}

The most common heuristic for the multidimensional dynamic program ming technique is the PPA (42), which is based on the landmark work by Feng and Doolittle (12). Several different implementations of this approach are available, but all are conceptually similar. A number of programs implementing the PPA algorithm have been developed, including GCG's PileUp (9), MULTALIGN (4), and ClustalW (49), and each performs a set of steps similar to those described in Table 2.

The PPAs are designed to have a

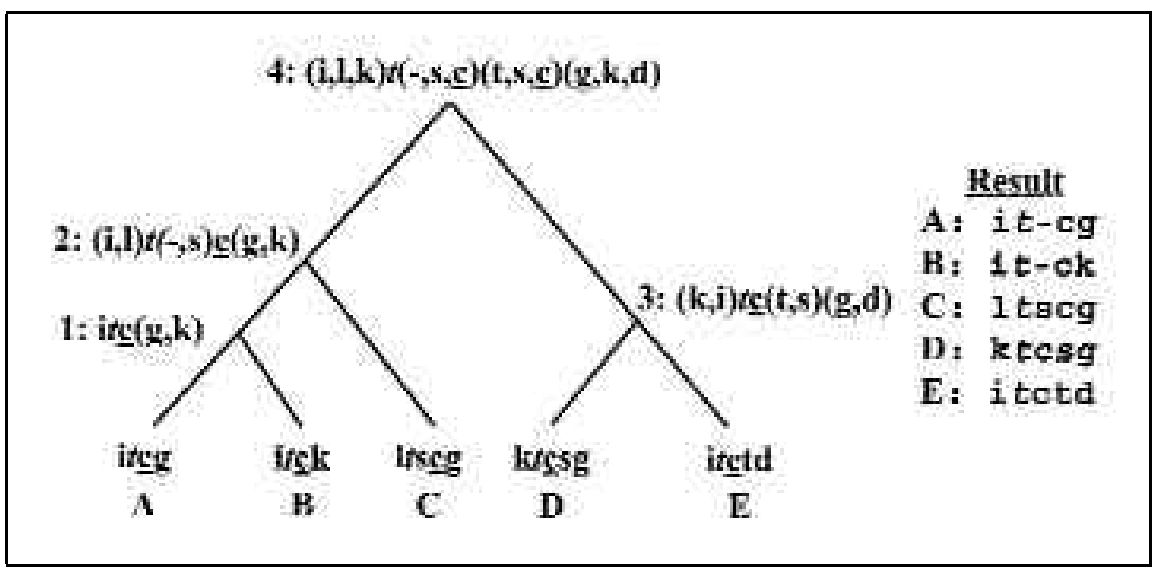

Figure 4. PPA of five sequences using a guide tree. The resulting alignment from this process is shown to the right. The conserved threonine $(t)$ is correctly aligned while the conserved cysteine (c) $)$ is not correctly aligned into a single column. A complete description of the alignment process is described in the text. 


\section{Review}

Table 2. The PPA Algorithm

\begin{tabular}{|c|c|}
\hline Step & Procedure Description \\
\hline 1. Pairwise Distances & $\begin{array}{l}\text { Perform Needleman-Wunsch (global) alignment }(30,44) \text { on all sequence pairs to find the distance } \\
\text { between all pairs of sequences. }\end{array}$ \\
\hline $\begin{array}{l}\text { 2. Cluster the } \\
\text { Pairwise Distances }\end{array}$ & $\begin{array}{l}\text { Perform a simple clustering to determine which pairs of sequences are closer than others. Moore et } \\
\text { al. (29) first reported using pairwise alignments iteratively to create phylogenetic relationships, } \\
\text { which then allows for the creation of either a UPGMA-constructed guide tree (48)a or a Neighbor- } \\
\text { Joining guide tree }(41)^{b} \text { (both rooted trees). These joining trees are based on alignment scores and } \\
\text { non-biological rules for creating trees; thus, they should be used cautiously as an evolutionary tree. } \\
\text { This step represents a major difference among the various implementations of the PPA and is the } \\
\text { part of the algorithm where some of the greatest improvements have occurred. }\end{array}$ \\
\hline $\begin{array}{l}\text { 3. Align the Sequences } \\
\text { Guided by the } \\
\text { Clustering }\end{array}$ & $\begin{array}{l}\text { Align the closest sequences in the joining tree together, followed by adding more sequences to } \\
\text { the initial alignment. For example, when using an UPGMA guide tree or Neighbor-Joining guide } \\
\text { tree, one would align a pair of sequences by starting at the bottom of a branch and successively } \\
\text { adding more sequences (Figure } 4 \text { ) to the nascent alignment (the nascent alignment defines the } \\
\text { range of possibilities for the ancestral sequence). Sequences A and B are aligned to give nascent } \\
\text { alignment } 1 \text {, which is then aligned with sequence } C \text { to give nascent alignment } 2 \text {. Sequences D and } \\
\text { E are aligned to give nascent alignment } 3 \text {, which is combined with nascent alignment } 2 \text { to give the fi- } \\
\text { nal alignment } 4 \text {. }\end{array}$ \\
\hline
\end{tabular}

This table outlines the steps of a generalized PPA algorithm used by many of the popular multiple sequence alignment programs.

aStarting with two sequences in a small tree (essentially a line), evaluate all possible orientations for the addition of a third sequence (off the center of the line, closer to the first sequence, or closer to the second sequence, plus the length of the branch from the original line to the third sequence) and choose the best combination. This process is repeated sequentially for all sequences. Finally, the tree is refined by a process of swapping adjacent branches in the search for better trees.

bThe Neighbor-Joining method (31) starts with all sequences in a star and then adjusts all of the branching points and branch lengths. The branch lengths are used to compute sequence weights, which are used during the alignment of the nascent sequence, with either a second nascent sequence or a sequence.

higher intrinsic regard for recent events as compared with distant ones. Thus, subregions are often of higher quality than the overall alignment, which can suffer from local minima problems caused by looking at only a subset of the data at any one time and the inability to change the alignment of sequences in early nascent alignments on the basis of sequences added subsequently. The most common error found in PPAs is the failure to identify highly conserved residues, which are placed into several columns of the alignment rather than into a single column. This problem can be clearly seen in the Result in Figure 4, where the cysteine (C) in each sequence is not aligned, but the conserved threonine (T) column was found. The addition of a pair of gaps into nascent alignment " 3 " would have allowed this to be found. ClustalW has added several ad hoc rules (49) to try to correct these problems and is usually rated as the best of the basic PPA algorithms.

The multidimensional dynamic programming technique will find the minimal distance path through the alignment space. This is compared with PPAs, which only enforce a local minimal distance criterion at each step, not a global one. Thus, the alignments from PPA will tend to have more, and perhaps substantially more, columns than the minimal distance techniques. Furthermore, the PPAs often have "hanging residues"- one or two residues centered in a gap or in a large number of small gaps in a poorly defined region. The free introduction of gaps can potentially allow spurious identification of "conserved residues" and the more common failure to identify residues that have been conserved during the evolution.

A number of programs attempt, with some success, to avoid the local minima problems associated with the PPA methodology while retaining its speed and ability to deal with large alignment problems. DbClustal (53) and T-Coffee (38) use a consistency analysis to bring additional information to and constrain the PPA technique (see previously described scoring methods). The ComA lign (7) program generates guide trees at random and computes many PPA alignments. ComAlign then computes a full sum-of-pairs score for each of these different PPA alignments and selects and joins the best scoring segments, contiguous groups of columns, to piece together in a final alignment that com bines the best features of several of the initial alignments.

The iterative techniques differ from the previously described approaches in that the multidimensional dynamic programming technique and the PPA each compute a single optimized result that represented the care taken during the creation of the alignment. Alternatively, one could construct a lower-quality 
alignment, followed by the refinement of the alignment, in a series of well-defined iterative steps. The iterative approaches tend to have modest memory requirements, while the computational time is usually greater than the PPA and can exceed the requirements of the multidimensional dynamic program ming technique.

The genetic algorithm (GA) is an optimization approach based on a stylized evolutionary model allowing for mutation-like and crossover events that has been applied to multiple sequence alignment (36). The GA search is based on the creation of a large enough number of alignments to allow a thorough search of the possible solutions. This approach is not as computationally efficient because prior knowledge (nascent alignments) is only partially used in the creation of the next generation of alignments. This feature also allows these programs to escape from local minima. It has been shown that GAs find the best alignment if they are run long enough. The GA algorithm can be described in a simple flowchart (Figure 5), with the iterative portion consisting of two main, decoupled components: the evaluation of the population of alignments to determine which members will be replaced, followed by the creation of the next generation of child alignments (36). Although the memory requirement of GAs is substantially less than the restricted-space multidimensional dynamic program ming algorithm, the CPU time requirements can be substantially greater. Overall, the results from a GA program, such as SAGA (36), can be virtually identical to the results obtained from the restricted-space multidimensional dynamic programming algorithm, MSA, but may fail to find a satisfactory alignment with highly diverged sequences. The decoupling of the scoring and alignment generation functions potentially allows the use of any scoring method, not just the sum-of-pairs method. Therefore, an intriguing possibility would be a GA program with a tree-based or traveling salesman scoring method. While not currently available, this program should be straightforward to develop.

The most recent version of SAGA (36) incorporates the ability to optimize an alignment based on a consistency measure called the consistence-based objective function for alignment evaluation (COFFEE) (37). This measure is an early, simpler version of the T-Coffee consistency scores. The COFFEE consistency measure optimized the number of aligned residues that were also aligned in global pairwise alignments of the same sequences. In some cases, the alignments generated using the COFFEE objective function were better than those found using the sumof-pairs objective function (37).

The PRRP (17) program optimizes a progressive pairwise, global alignment by iteratively dividing the sequences into two groups. Subsequently, these groups are realigned using a global group-to-group alignment algorithm until an optimal multiple sequence alignment is obtained.

MULTALIN (8) performs the same initial distance computation as PPAs, followed by hierarchical clustering. The hierarchical clustering is then used to create an initial multiple sequence alignment in a process similar to the PPAs. MULTALIN then recomputes the distance for each pairwise alignment using the extracted pairwise alignment from the multiple sequence alignment and then uses these new distances to create a new set of hierarchical clusters that ultimately provide the next multiple sequence alignment. This process continues iterating until the alignment cannot be further optimized. Gotoh (18) has shown that a random partition will converge to the optimal sum-of-pairs alignment.

HMMs are an attractive approach to multiple sequence alignment because they are on a more solid statistical footing than the previously described classical approaches $(3,11)$. HMMs are fully grounded in probability theory; neither the phylogenic history nor the pairwise distances are required, and gaps are treated in a variable, position-dependent manner. HMMs assume that each sequence and every residue within the sequences are independent of each other, assumptions clearly violated by the evolutionary origins of biological sequences. For each position in the alignment, one must compute the probability of a main (match or mismatch) insertion or deletion state, with a probability for the occurrence of each residue in the main and insertion states. This results in 
almost 50 probabilities that must be computed for each alignment position, which represents the greatest flexibility and number of parameters for any of the approaches. A number of training methods have been implemented for estimating these probabilities from the sequences used to build the HMM. Perhaps, because of the flexibility and resulting large number of parameters to be estimated, the ability to routinely obtain high-quality multiple sequence alignments from HMMs using these training methods has proven to be difficult and generally requires considerable expertise and careful attention to each creation of an HMM from unaligned sequences (27). Instead of training the HMM from unaligned sequences, HMMs are frequently created directly from high-quality multiple sequence alignments that were generated by other means. HMMs are a powerful statistical representation of a family of sequences and can be used effectively to identify highly diverged homologues and to create an alignment that includes new fam ily members by aligning all of the sequences to the HMM. Their most widespread use is in libraries of protein families such as Pfam (5).

\section{ADDITIONAL ALIGNMENT DETAILS}

Ultimately, regardless of the scoring method or implementation selected, one must have a quantitative model for the cost to create a gap and the replacement of a residue. The following section describes the gap penalty and distance measure used in a number of implementations for the sum-of-pairs scoring method.

\section{Gap Penalty}

The previously described programs implement an affine gap model, which consists of a penalty for opening and then extending the gap. The affine gap model is also used by the commonly used pairwise alignment programs (34), with the difference being that the terms are specified as distances or costs for the multiple sequence alignment, while the terms are expressed as similarities for the pairwise alignments. Plus, there are additional issues that arise in the context of gap costs for multiple sequence alignments.

Altschul (1) has pointed out that the cost for insertion and deletion events (gap costs) in multiple sequence alignments should have a common rationale, with the substitution costs used in the multiple sequence alignment procedure. Altschul proposed that multiple sequence alignments that were evaluated by sum-of-pairs substitution costs should have gap costs that were equal to the sum of the gap costs in the pairwise alignments implied by the multiple sequence alignment. Gap costs computed this way are generally referred to as "natural gap costs".

Unfortunately, computing natural gap costs while simultaneously generating and evaluating a multiple sequence alignment step by step requires that a program retain an enormous amount of information about the calculations leading up to the point currently being evaluated. This amount of information is too large to carry out the natural gap cost calculation in programs that build up and evaluate a multiple sequence alignment step by step. Thus, the SAGA program is the only one of the programs discussed here that allows the use of natural gap cost. This is because computing natural gap costs for a completed multiple sequence alignment, as SAGA does, is both fast and straightforward.

The MSA program, which generates and evaluates a multiple sequence alignment step by step using a sum-ofpairs scoring scheme, uses what are called quasi-natural gap costs (1). Quasi-natural gap costs require the program using them to carry along only a very minimal amount of the history of the calculation leading up to the current step in the procedure and differ from the natural gap costs only in a single, relatively rare circumstance. Thus, the quasi-natural gap costs will be one open gap penalty larger than the natural gap costs in the following situation. An implied pairwise alignment must contain a section that has gap characters in one sequence aligned with gap characters in the second sequence. Addition-

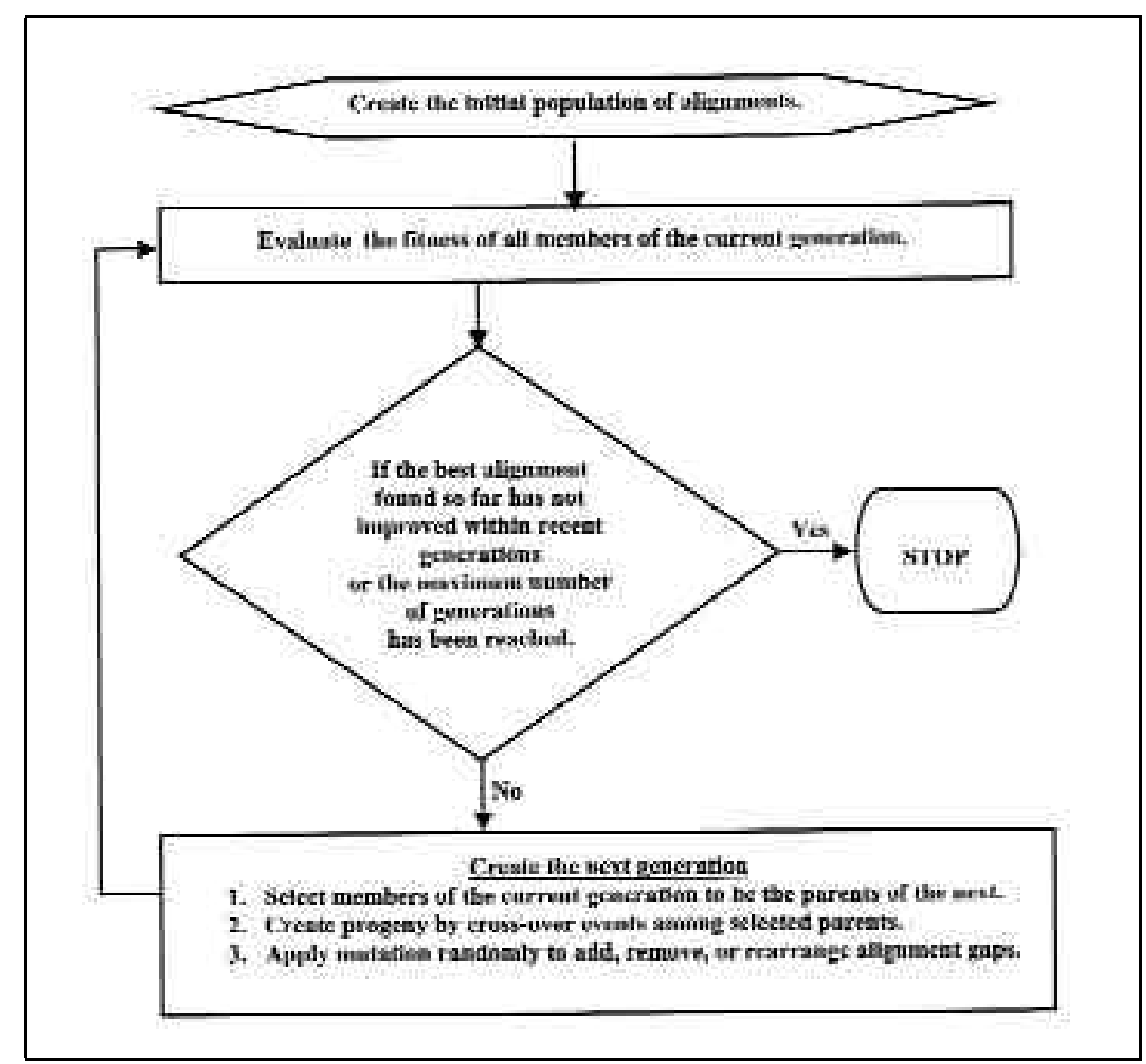

Figure 5. Flowchart that describes the GA approach to multiple sequence alignment. 
ally, the run of gap characters in one of the sequences must both begin after and end before the run of gap characters in the other sequence of the implied pairwise alignment.

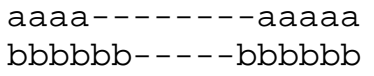

The PPA programs such as ClustalW or PileUp accumulate and charge for gaps as each sequence or previously aligned group of sequences is merged with another sequence or previously aligned group of sequences. This is carried out under a rule named “once a gap, always a gap". Under this rule, the only allowed change in an already existing gap is its extension by introducing new gap characters into the gap. This results from introducing a new gap into all of the aligned sequences that had been aligned in previous stages. For example, the already aligned $a$ and $b$ sequences could be aligned with the $c$ sequence to generate new gap characters.

aaaa----aaaaa

b.bbbbbbbbbb.bbb

This could be aligned with the $\mathrm{c}$ sequence to yield:

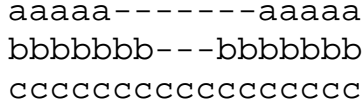

The treatment of gaps in a sum-ofpairs model is consistent with the scoring of normal sequence residues. Many of its limitations derive from the fact that in the sum-of-pairs model a gap character is treated similarly to an additional kind of sequence residue, rather than as the result of a unique evolutionary event, an insertion or deletion.

The ClustalW program (49) attempts (and generally succeeds) to refine this procedure by implementing a series of heuristic rules about where gaps are more likely to be introduced (50). However, while these heuristic rules generally work well, like any heuristic rule, they can sometimes be counterproductive. The main heuristic rules make it more likely that gap characters will be introduced where they align with gap characters introduced in previous steps and that it will be less likely to introduce completely new gaps within eight characters of an al- ready existing gap. Gaps are also more likely to be introduced into a run of five or more hydrophilic amino acids (which is taken to imply a high likelihood of a surface loop in the protein). When none of the above heuristic rules are operative, each amino acid is assigned an individual likelihood of having a gap introduced between it and the next amino acid in the c-terminal direction along the sequence. The user has some control over how these rules are used in running the ClustalW program.

The T-Coffee procedure (38) does not use gap penalties, which means that both the open gap and extend gap penalties are set to zero in the final PPA phase. However, non-zero gap penalties are used in computing both the global and local alignments that are used to calculate the consistency scores used in the final PPA phase. Thus, the penalties from the pairwise alignment phase are implicit in the consistency scores. Additionally, the consistency scores tend to place such a strong emphasis on aligning the highly conserved regions among the sequences that this feature dominates the process of allocating alignment gaps.

\section{DISTANCE MEASURE}

While most widely used pairwise alignment programs present alignment scores in terms of similarity between residues (34), most multiple sequence alignment programs use a distance measure (sometimes referred to as a cost) to compute an alignment score. Distances have properties that allow sensible discussions of the simultaneous relationship between three or more objects, such as sequences (23), while similarity measures generally only permit the simultaneous consideration of relationships between pairs of objects. Specifically, distances allow the construction of maps, which are a representation of the simultaneous relationships among multiple objects. The property of distances that is most critical for creating maps is that distances obey the triangle inequality, which is an algebraic statement of the Euclidian postulate that three sides determine a triangle. Thus, from this perspective, a multiple sequence alignment can be 
thought of as an unconventional map.

The distance measures used in multiple sequence alignment are generally derived from the similarity measures used in pairwise alignment and database searching and are most often referred to by the same name (e.g., PAM250). The conversion is usually performed using the formula described by Smith et al. (47) to provide for the interconversion of distance and similarity measures to yield identical pairwise alignments using both measures.

\section{DISCUSSION}

Residues within a family's multiple sequence alignment can be categorized into one of three classes: residues that are highly conserved over the entire superfamily (Class I); residues that are highly variable over the entire superfamily (Class II); and residues that show highly limited variability within one or a few families encompassed by the aligned superfamily (Class III).
Class I residues usually serve in a key structural or functional role across all of the sequences, while Class III residues may be critical to the different physiologic roles that the different paralogous sequences fulfill. Thus, the identification of the Class III residues may identify residues responsible for selectivity, specificity, or interaction sites that vary between paralogous sequences. If your primary goal is to identify the majority of the Class I residues, then almost any of the automatic Web-based techniques (14) will provide an acceptable answer. If the goal is to identify all conserved residues and some conserved patterns, then a PPA will need to be edited using additional information, such as the results of an MSA (25) alignment or conserved patterns from a local pattern program $(2,6,24)$. Only a high-quality multiple sequence alignment $(26,39,51$, 52 ) will allow for the accurate differentiation between randomly mutated residues (Class II) and those residues potentially responsible for selectivity or specificity (Class III).

In general, any automatically produced alignment can be improved (26, $39,51,52)$ and, given the proper set of tools, should be. There are a number of tools available to assist in editing the initial multiple sequence alignment. This includes a number of alignment editors [e.g., GeneDoc (http://www.psc. edu/biomed/genedoc), LineUp (9), and SeaView (13)], programs to identify conserved patterns [e.g., MEME (2) and the Gibbs Sampler (24)], and methods that describe the robustness of pairwise alignments and thus provide information about the regions within the multiple sequence alignment that are most likely to vary $(20,58)$. These topics are beyond the scope of this paper and will be addressed in a future publication.

A number of studies $(14,22,26,33$, 51,52 ) have evaluated the numerous multiple sequence alignment programs. All of the studies we have cited have been limited to programs using the sumof-pairs scoring method. A few generalizations can be drawn from these studies. The MSA program is routinely the best and provides the correct answer for modest problems, but MSA's computational requirements limit the size of the problems that it can be applied to.
ClustalW is usually rated as the best of the PPA programs. Two of the iterative programs, SAGA and PRRP, provide good results but can require substantially more CPU time than the heuristics $(17,18,36,52)$. HMMs and their associated machine-learning training methods $(11,27)$ have not proven to be as effective a tool for creating multiple sequence alignments as had initially been expected, at least when applied by inexperienced users. At this time, inexperienced users from the biology community should probably use some other method to align sequences and then create an HMM from the alignment. The use of HMMs to represent a multiple sequence alignment and perform additional analyses can be worthwhile and is recommended.

Some of the newer alignment programs discussed in this paper [i.e., ComAlign (7), DbClustal (53), and TCoffee (38)] were not included in the comprehensive reviews cited above because they are too recent. However, the examples presented by their originators make us hopeful that they represent a substantial improvement in the capabilities of multiple sequence alignment programs. In particular, on a small number of protein sequence families that have proven to have regions that PPAs such as ClustalW have trouble aligning, we have obtained superior results from the T-Coffee (38) program. In addition, the operative gap function of T-Coffee allowed it to find solutions that were superior, in terms of corresponding to structural superposition results, to those found by the rigorous maximizing of the gap function (1) by the MSA (25) program. A problem with 153 protein sequences averaging about 220 amino acids in length that took six minutes of computer processor time for ClustalW took well over two days of computer processor time for TCoffee. This problem would require too much computer time and memory to have been solved by MSA on any com puter. Thus, this improvement comes at the cost of a significant increase in computer resources for T-Coffee over ClustalW and other PPA programs.

All generally available multiple sequence alignment programs have theoretical and practical limitations. A few generalizations can be made about these 
limitations. All of the programs in widespread use are theoretically limited by not optimizing the alignment over a rigorous evolutionary model. Instead, they optimize over a surrogate measure that optimizes some measure of conservation. Because highly conserved sequence residues are the primary goal of multiple sequence alignment and are an expected result of sequence evolution, these surrogates have worked well, and new developments are making them even better. However, it is highly likely that as these surrogates become more rigorous and effective, they will yield alignments that show some spurious conservation. This spurious conservation will most likely show up in the juxtaposition of alignment gaps that result from insertion and deletion events that occurred independently (in different parts of the phylogenetic tree) at slightly different locations in the sequences. PPA programs have sporadically tended to miss highly conserved residues that more rigorous sum-of-pairs optimization would have correctly identified. Recent developments $(7,38,53)$ seem to be greatly reducing this artifact. The length of the alignment from a PPA will tend to be longer than from alignments generated by more rigorous sum-of-pairs optimization because they are less forceful about aligning gaps with each other into fewer columns of the alignment. The lack of rigorous optimization also leads PPA programs to have hanging residues with a large number of gaps in poorly defined regions. The iterative programs will, with a large number of iterations, approach the alignment found with the multidimensional dynamic program ming technique. However, the iterative programs can suffer from convergence problems, particularly when faced with highly diverged sequences.

\section{Future Directions}

The research into improved multiple sequence alignments is currently focusing on several different aspects rather than being narrowly focused $(7,38,40$, 53). These include better scoring functions $(16,37,38)$, using supplemental information to improve alignment results (53), and better algorithms for rigorous optimization (40). We expect research to continue in all of these areas.
For practical widespread use by practicing molecular biologists and biochemists, we expect the development of better scoring functions that incorporate supplementary information to provide the most near-term improvement $(7,37,38,53)$. The most recent developments in these areas seem to have resulted in some fairly significant practical improvements. These improvements are recent enough that final judgment should await more widespread testing and evaluation of these new techniques. As previously described (38), being able to recognize the circumstances that are likely to have produced a faulty alignment is becoming a critical need.

We believe that in the long term laboratory researchers will generally not create multiple sequence alignments from scratch to inform their research. Creating a multiple sequence alignment from scratch will be necessary only when a completely new gene or protein family is discovered. Given the number of complete genome sequencing projects being undertaken, this will become very rare. Increasingly, researchers will be able to search a database of protein family alignments, as is widely done now with Pfam (5). The database will be linked or integrated with a wide variety of genetic, biochemical, physiological, and structural information along the lines of the iProClass database (57). The family alignments themselves will be expertly crafted and carefully refined, as in now being done with BaliBase (51). Users will be able to integrate easily new sequences they have determined into their personal copy of the alignment, with the assurance that the overall alignment is of high quality and a useful guide to their laboratory research.

\section{ACKNOWLEDGMENTS}

This tutorial was developed from lectures that were presented as a part of workshops and courses funded by the National Institutes of Health NCRR grant no. P41 RR06009 and NHGRI grant no. T15 HG00015 and the HHMI grant to the University of Pittsburgh and the University of California at Davis. We thank the former students in the courses that we have taught, along with the other instructors, including Gary Churchill (Jackson Laboratory), Mike Gribskov (SDSC), and Steven Altschul (NLM), from whose lectures we have benefited greatly. Finally, we thank the referees, whose suggestions markedly improved the paper.

\section{REFERENCES}

1.Altschul, S.F. 1989. Gap costs for multiple sequence alignment. J. Theor. Biol. 138:297309.

2.Bailey, T.L. and C. Elkan. 1994. Fitting a mixture model by expectation maximization to discover motifs in biopolymers, p. 28-36. In R. Altman, D. Brutag, P. Karp, R. Lathrop, and D. Searls (Eds.), Proceedings of the Second International Conference on Intelligent Systems for Molecular Biology. AAAI Press, Menlo Park, CA.

3.Baldi, P., Y. Chauvin, T. Hunkapiller, and M.A. McClure. 1994. Hidden Markov models of biological primary sequence information. Proc. Natl. Acad. Sci. USA 91:10591062.

4.Barton, G.J. and M.J.E. Sternberg. 1987. A strategy for the rapid multiple alignment of proteins sequences-confidence levels from tertiary structure comparisons. J. Mol. Biol. 198:327-337.

5.Bateman, A., E. Birney, R. Durbin, S.R. Eddy, K.L. Howe, and E.L.L. Sonnham mer. 2000. The Pfam protein families database. Nucleic Acids Res. 28:263-266.

6.Brocchieri, L. and S. Karlin. 1998. A sym metric-iterated multiple alignment of protein sequences. J. Mol. Biol. 276:249-264.

7.Bucka-Lassen, K., O. Caprani, and J. Hein. 1999. Combining many multiple alignments in one improved alignment. Bioinformatics 15:122-130.

8.Corpet, F. 1988. Multiple sequence alignment with hierarchical-clustering. Nucleic Acids Res. 16:37-40.

9.Devereux, J., P. Haeberli, and O. Smithies. 1984. A comprehensive set of sequence analysis programs for the VAX. Nucleic Acids Res. 12:387-395.

10.Durbin, R., S.R. Eddy, A. Krough, and G. Mitchison. 1998. Biological Sequence Analysis: Probabilistic Models of Protein and Nucleic Acids. Cambridge University Press, Cambridge, UK.

11.Eddy, S.R. 1995. Multiple alignment using hidden Markov models. Proc. Int. Conf. Intell. Syst. Mol. Biol. 3:114-120.

12.Feng, D.-F. and R.F. Doolittle. 1987. Progressive sequence alignment as a prerequisite to correct phylogenetic trees. J. Mol. Evol. 25: 351-360.

13.Galtier, N., M. Gouy, and C. Gautier. 1996. SeaView and Phylo_win, two graphic tools for sequence alignment and molecular phylogeny. Comput. Appl. Biosci. 12:543-548.

14.Gaskell, G.J. 2000. Multiple sequence alignment tools on the Web. BioTechniques 29:6062. 
15.Gonnet, G.H., M.T. Hallett, C. Korostensky, and L. Bernardin. 2000. Darwin v. 2.0: an interpreted computer language for the biosciences. Bioinformatics 16:101-103.

16.Gonnet, G.H., C. Korostensky, and S. Benner. 2000. Evaluation measures of multiple sequence alignments. J. Comput. Biol. 7:261276.

17.Gotoh, O. 1996. Significant improvement in accuracy of multiple protein sequence alignments by iterative refinement as assessed by reference to structural alignments. J. Mol. Biol. 264:823-838.

18.Gotoh, O. 1999. Multiple sequence alignment: algorithms and applications. Adv. Biophys. 36:159-206.

19.Gupta, S.K., J.D. Kececioglu, and A.A Schaffer. 1995. Making the shortest-paths approach to sum-of-pairs multiple sequence alignment more space efficient in practice. J. Comput. Biol. 2:459-472.

20.Gusfield, D. and P. Stelling. 1996. Parametric and inverse-parametric sequence alignment with XPARAL. Methods Enzymol. 266:481-494.

21.Hein, J. 1990. Unified approach to alignment and phylogenies. Methods Enzymol. 183:626645.

22.Hirosawa, M., Y. Totoki, M. Hoshida, and M. Ishikawa. 1995. Comprehensive study on iterative algorithms of multiple sequence alignment. Comput. Appl. Biosci. 11:13-18.

23.Kruskal, J.B. 1999. An overview of sequence comparison, p. 1-44. In D. Sankoff and J. Kruskal (Eds.), Time Warps, String Edits, and Macromolecules. CSLI Publications, Stanford, CA.

24.Lawrence, C.E., S.F. Altschul, M.S. Boguski, J.S. Lui, A.F. Neuwald, and J.C. Wootton. 1993. Detecting subtle sequence signals: a Gibbs sampling strategy for multiple alignment. Science 262:208-214.

25.Lipman, D.J., S.F. Altschul, and J.D. Kececioglu. 1989. A tool for multiple sequence alignment. Proc. Natl. Acad. Sci. USA 86: 4412-4415.

26.McClure, M.A., T.K. Vasi, and W.M. Fitch. 1994. Comparative analysis of multiple protein-sequence alignment methods. Mol. Biol. Evol. 11:571-592.

27.McClure, M.A., C. Smith, and P. Elton. 1996. Parameterization studies for the SAM and HMMER methods of hidden Markov model generation, p. 155-164. In D.J. States, P. Agarwal, T. Gaasterland, L. Hunter, and R.F. Smith (Eds.), Proceedings, Fourth International Conference on Intelligent Systems for Molecular Biology. AAAI Press, Menlo Park, CA.

28.Moens, L., J. Vanfleteren, Y. Van de Peer, K. Peeters, O. Kapp, J. Czeluzniak, M. Goodman, M. Blaxter et al. 1996. Globins in nonvertebrate species: dispersal by horizontal gene transfer and evolution of the structurefunction relationships. Mol. Biol. Evol. 13:324-333.

29.Moore, G.M., M. Goodman, and J. Barnabas. 1973. Iterative approach from standpoint of additive hypothesis of dendrogram problem posed by molecular data sets. J. Theor. Biol. 38:423-457.

30.Needleman, S.B. and C.D. Wunsch. 1970. A general method applicable to the search for similarities in the amino acid sequences of two proteins. J. Mol. Biol. 48:443-453.

31.Nei, M. 1996. Phylogenetic analysis in molecular evolutionary genetics. Annu. Rev. Genet. 30:371-403.

32.Nicholas, H.B., Jr., B. Persson, H. Jornvall, and J. Hempel. 1995. Ethanol utilization regulatory protein: profile alignments give no evidence of origin through aldehyde and alcohol dehydrogenase gene fusion. Protein Sci. 4:2621-2624.

33.Nicholas, H.B., Jr., A.J. Ropelewski, D.W. Deerfield II, and J.G. Behrmann. 1995. A metric measure for comparing sequence alignments in methods in protein sequence analysis, p. 515-525. In M.Z. Atassi and E. Appella (Eds.), Proceedings of the 10th International Conference on Methods in Protein Structure. Plenum Press, New York.

34.Nicholas, H.B., Jr., D.W. Deerfield II, and A.J. Ropelewski. 2000. Strategies for searching sequence databases. BioTechniques 28: 1174-1191.

35.Nicholas, H.B., Jr., H.N. Arst, Jr., and M.X. Caddick. 2001. Evaluating low level sequence identities. Are Aspergillus QUTA and AROM homologous? Eur. J. Biochem. 268:414-419.

36.Notredame, C. and D.G. Higgins. 1996. SAGA: sequence alignment by genetic algorithm. Nucleic Acids Res. 24:1515-1524.

37.Notredame, C., L. Holm, and D.G. Higgins. 1998. COFFEE: an objective function for multiple sequence alignments. Bioinformatics 14:407-422.

38.Notredame, C., D.G. Higgins, and J. Heringa. 2000. T-Coffee: a novel method for fast and accurate multiple sequence alignment. J. Mol. Biol. 302:205-217.

39.Plewniak, F., J.D. Thompson, and O. Poch. 2000. Ballast: Blast post-processing based on locally conserved segments. Bioinformatics 16:750-759.

40.Reinert, K., J. Stoye, and T. Will. 2000. An iterative method for faster sum-of-pairs multiple sequence alignment. Bioinformatics 16: 808-814

41.Saitou, N. and M. Nei. 1987. The neighborjoining method-a new method for reconstructing phylogenetic trees. Mol. Biol. Evol. 4:406-425.

42.Sankoff, D. 1975. Minimal mutation trees of sequences. SIAM J. Appl. Math. 28:35-42.

43.Sankoff, D. and R.J. Cedergren. 1999. Simultaneous comparison of three or more sequences related by a tree, p. 253-263. In D. Sankoff and J. Kruskal (Eds.), Time Warps, String Edits, and Macromolecules. CSLI Publications, Stanford, CA.

44.Sellers, P.H. 1974. On the theory and computation of evolutionary distances. SIAM J. Appl. Math. 26:787-793.

45.Sellers, P.H. 1979. Pattern recognition in genetic sequences. Proc. Natl. Acad. Sci. USA 76:3041-3041.

46.Smith, T.F. and M.S. Waterman. 1981. Identification of common molecular subsequences. J. Mol. Biol. 147:195-197.

47.Smith, T.F., M.S. Waterman, and W.M. Fitch. 1981. Comparative biosequence metrics. J. Mol. Evol. 18:38-46.
48.Sneath, P.H. and R.R. Sokal. 1973. Numerical Taxonomy. Freeman Publishing, San Francisco.

49.Thompson, J.D., D.G. Higgins, and T.J. Gibson. 1994. ClustalW: improving the sensitivity of progressive multiple sequence alignment through sequence weighting, positionspecific gap penalties, and weight matrix choice. Nucleic Acids Res. 22:4673-4680.

50.Thompson, J.D. 1995. Introducing variable gap penalties to sequence alignments in linear space. Comput. Appl. Biosci. 11:181-186.

51.Thompson, J.D., F. Plewniak, and O. Poch. 1999. BaliBase: a benchmark alignment database for the evaluation of multiple sequence alignment programs. Bioinformatics 15:8788

52.Thompson, J.D., F. Plewniak, and O. Poch. 1999. A comprehensive comparison of multiple sequence alignment programs. Nucleic Acids Res. 27:2682-2690.

53.Thompson, J.D., F. Plewniak, J.-C. Thierry, and O. Poch. 2000. DbClustal: rapid and reliable global multiple alignments of protein sequences detected by database searches. Nucleic Acids Res. 28:2919-2926.

54. Wheeler, W.C. and D.S. Gladstein. 1994 MALIGN: a multiple sequence alignment program. J. Heredity, 85:417-418.

55.Whiting, M.F., J.C. Carpenter, Q.D. Wheeler, and W.C. Wheeler. 1997. The strepsiptera problem: phylogeny of the holometabolous insect orders inferred from $18 \mathrm{~S}$ and $28 \mathrm{~S}$ ribosomal DNA sequences and morphology. Syst. Biol. 46:1-68.

56. Wang, L.S., T. Jiang, and D. Gusfield. 2000. A more efficient approximation scheme for tree alignment. SIAM J. Comput. 30:283-299.

57.Wu, C., C. Xiao, Z. Hou, H. Huang, and W.C. Barker. 2001. iProClass: an integrated, comprehensive, and annotated protein classification database. Nucleic Acids Res. 29:52-54.

58.Zucker, M. 1991. Suboptimal sequence alignment in molecular biology: alignment with error analysis. J. Mol. Biol. 221:403-420.

\author{
Address correspondence to: \\ Dr. Hugh B. Nicholas Jr. \\ Pittsburgh Supercomputing Center \\ Carnegie Mellon University \\ 4400 Fifth Avenue \\ Pittsburgh, PA 15213, USA \\ e-mail:nicholas@psc.edu
}

For reprints of this or any other article, contact Reprints@BioTechniques.com 